\title{
Overlap syndrome between chronic obstructive pulmonary disease and obstructive sleep apnoea in a Southeast Asian teaching hospital
}

Sridhar $\underline{\text { Venkateswaran }}^{1}$, MBBS, FRACP, Augustine $\underline{\text { Tee }}^{1}$, MBBS, MRCP

INTRODUCTION Overlap syndrome between obstructive sleep apnoea (OSA) and chronic obstructive pulmonary disease (COPD) is important but under-recognised. We aimed to determine the prevalence of overlap syndrome and the predictors of OSA in patients with COPD.

METHODS Patients aged $\geq 40$ years were recruited from a dedicated COPD clinic and underwent overnight polysomnography. A diagnosis of OSA was made when apnoea-hypopnoea index (AHI) was $\geq 5$.

RESULTS In all, 22 patients (aged $71 \pm 9$ years), predominantly men, were recruited. Mean values recorded were: predicted forced expiratory volume in the first second percentage $55 \pm 15$; body mass index $23.7 \pm 6.5 \mathrm{~kg} / \mathrm{m}^{2}$; Epworth Sleepiness Scale score $5.6 \pm 5.8$; and AHI $15.8 \pm 18.6$. Among the 14 patients with OSA (prevalence of overlap syndrome at $63.6 \%$ ), the mean number of hospital visits for COPD exacerbations in the preceding one year was $0.5 \pm 0.7$. Patients with overlap syndrome had worse modified Medical Research Council dyspnoea scale scores and a lower percentage of rapid eye movement (REM) sleep than patients without. There were no other statistical differences in lung function or sleep study indices between the two patient groups.

CONCLUSION The majority of our patients had overlap syndrome and minimal exacerbations, and were not obese or sleepy. Significant differences between patients with and without overlap syndrome were seen in two aspects - the former was more dyspnoeic and had less REM sleep. Our findings suggest that standard clinical predictors cannot be used for patients with overlap syndrome, and therefore, a high index of suspicion is needed.

Keywords: chronic obstructive pulmonary disease, obstructive sleep apnoea, overlap syndrome

\section{INTRODUCTION}

Coined by Flenley, the term 'overlap syndrome' describes the coexistence of two diseases - obstructive sleep apnoea (OSA) and chronic obstructive pulmonary disease (COPD). ${ }^{(1)}$ While OSA and COPD each carries its own comorbidities and complications, it has been surmised that patients with overlap syndrome may have a worse prognosis than patients with only one of either disease..$^{(2)}$ Taking into account the individual prevalences of COPD and OSA, it has also been suggested that the prevalence of overlap syndrome in adults aged 40 years and over is $0.5 \%-1 \%$. $^{(3-5)}$

To the best of our knowledge, no study in the literature has specifically investigated overlap syndrome in Asian patients. Therefore, the aim of the present study was to ascertain the prevalence of overlap syndrome, and examine the predictors (if any) and polysomnographic profile of OSA in patients with COPD at a Southeast Asian teaching hospital.

\section{METHODS}

Conducted between June 2009 and December 2010, this prospective study comprised adult patients aged 40 years and over who were recruited from a dedicated COPD clinic at the Department of Respiratory and Critical Care Medicine, Changi General Hospital, Singapore. All patients who were screened at the centre were recruited. There was only one dropout in our cohort. All patients were diagnosed as having COPD based on the
Global Initiative for Chronic Obstructive Lung Disease (GOLD) definition and spirometric criteria. ${ }^{(6)}$ In addition to the published definition of COPD, the other inclusion criterion was a smoking history (either current or previous) $\geq 30$ pack-years. Patients were excluded from the study if they had other chronic respiratory diseases such as asthma, bronchiectasis or pulmonary fibrosis, or known sleep-disordered breathing (SDB) such as OSA (as defined by the respiratory disturbance index [RDI] score $\geq 5$, with or without symptoms) and/or obesity hypoventilation syndrome (OHS). Patients were also excluded if they were on long-term oxygen therapy, and if body mass index (BMI) $\geq 30 \mathrm{~kg} / \mathrm{m}^{2}$.

Baseline demographic and anthropometric data were collected for all recruited patients. The Epworth Sleepiness Scale (ESS) was used to assess daytime sleepiness. ${ }^{(7)}$ Patients underwent an attended full-montage, multichannel, overnight diagnostic polysomnography using the Compumedics ProFusion Sleep 3 system (Compumedics Limited, Abbotsford, Victoria, Australia). All patients were followed up in the outpatient clinic a few weeks after the sleep study was completed. While our study was not designed to investigate the treatment of overlap syndrome, all recruited patients were treated for each individual disease based on the severity of the disease via positive airway pressure therapy for OSA and/or inhaled therapy for COPD. The present study was approved by the SingHealth Institutional Review Board. Written informed consent was obtained from all patients prior to the study.

${ }^{1}$ Department of Respiratory and Critical Care Medicine, Changi General Hospital, Singapore

Correspondence: Dr Sridhar Venkateswaran, Visiting Consultant, Department of Respiratory and Critical Care Medicine, Changi General Hospital, 2 Simei Street 3, Singapore 529889. sridhar_venkateswaran@cgh.com.sg. 
Characteristics of patients were presented as mean \pm standard deviation, where appropriate. Fisher's exact test was used for categorical variables and independent sample $t$-test was used for continuous variables to compare patients with overlap syndrome against those without. The predictors of OSA were not based on linear regression models. The 95\% confidence intervals $(\mathrm{Cls})$ were quoted, where appropriate. All analyses were performed using the Statistical Package for the Social Sciences version 19.0 (SPSS Inc, Chicago, IL, USA). A p-value $<0.05$ was considered statistically significant.

\section{RESULTS}

Table I presents the overall demographic characteristics of our study population $(n=22)$. In all, 23 patients were recruited for the study, with only one dropout. The prevalence of overlap syndrome in our cohort was 63.6\% (95\% Cl 43.0-80.3).

Tables II and III respectively present data pertaining to lung function and polysomnography of the study group. The proportion of patients classified as GOLD stages I-IV, based on their COPD severity, is also shown. The predicted mean forced expiratory volume in the first second $\left(\mathrm{FEV}_{1}\right)$ percentage placed a majority of our patients in GOLD stage II. The mean apnoeahypopnoea index $(\mathrm{AHI})^{(8)}$ indicated that the severity of OSA was moderate in our cohort. Patients with conditions ranging from no OSA to severe OSA were well represented. Sleep efficiency and percentage of rapid eye movement (REM) sleep were found to be reduced in our patients, while the arousal index $(\mathrm{AI})^{(9)}$ and periodic limb movement index (PLMI) ${ }^{(10)}$ were increased. The mean ESS score of our study cohort was within normal limits.

Table IV shows the COPD severity indices. The average distance walked in 6 min (6MWD) and the mean BODE (body mass index, airflow obstruction, dyspnea, and exercise) index ${ }^{(11)}$ reflected the severity of COPD in our cohort. Mean exacerbation rate for COPD in the preceding year was 0.5 , with 9 out of the 22 patients $(40.9 \%)$ having had at least one exacerbation during this period.

There was no difference between the patient groups with and without overlap syndrome, with regard to variables such as age, gender, ethnicity, BMI, FEV ${ }_{1}$, Medical Research Council (MRC) dyspnoea scale scores, BODE index, 6MWD, smoking (pack-years) and exacerbations in the preceding year $(p>0.05)$. However, where polysomnography variables were concerned, there were significant differences in percentage of REM (REM\%) sleep ( $p=0.04$; Fig. 1$)$ and Al score ( $p=0.03$; Fig. 2$)$ between the two patient groups.

Apart from GOLD stage II, where there were significantly less patients with overlap syndrome than those without, all the other GOLD stages only had patients with overlap syndrome ( $p=0.006$; Fig. 3$)$. There were no patients in GOLD stage IV. In other words, all patients with COPD in our study who were categorised as GOLD stage II did not have overlap syndrome.

\section{DISCUSSION}

The prevalence of overlap syndrome in our study population was $63.6 \%$. Such high incidence not only does not correlate with the real-world prevalence of overlap syndrome, ${ }^{(12)}$ but is also rather
Table I. Demographic characteristics of patients $(n=22)$.

\begin{tabular}{lc}
\hline Characteristic & No. (\%) \\
\hline Age* $\left.^{*} \mathbf{y r}\right)$ & $71 \pm 9$ \\
Male gender & $19(86)$ \\
Body mass index* (kg/m²) & $23.7 \pm 6.5$ \\
Smoking history* (pack-years) & $59 \pm 53$ \\
Ethnicity & \\
Chinese & $15(68.2)$ \\
Malay & $4(18.2)$ \\
Indian & $2(9.1)$ \\
Eurasian & $0(0.0)$ \\
Other & $1(4.5)$ \\
\hline
\end{tabular}

* Data is presented as mean \pm standard deviation

Table II. Lung function variables.

\begin{tabular}{|c|c|}
\hline Variable & No. (\%) \\
\hline FEV $_{1}^{*}(\mathrm{~L})$ & $1.2 \pm 0.4$ \\
\hline Predicted FEV ${ }_{1}{ }^{*}(\%)$ & $55 \pm 15$ \\
\hline \multicolumn{2}{|l|}{ Severity of COPD } \\
\hline 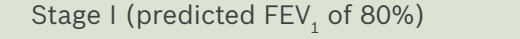 & $2(9.1)$ \\
\hline Stage II (predicted $\mathrm{FEV}_{1}$ of $50 \%-79 \%$ ) & $13(54.6)$ \\
\hline 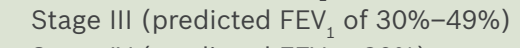 & $7(31.8)$ \\
\hline Stage IV (predicted FEV F $_{1} 30 \%$ ) & $0(0)$ \\
\hline Bronchodilator reversibility* (mL; \%) & $126 \pm 79 ; 6.6 \pm 8.5$ \\
\hline
\end{tabular}

* Data is presented as mean \pm standard deviation. COPD: chronic obstructive pulmonary disease; $\mathrm{FEV}_{1}$ : forced expiratory volume in the first second

Table III. Polysomnography variables.

\begin{tabular}{lc}
\hline Variable & Mean \pm SD \\
\hline Sleep efficiency (\%) & $63.6 \pm 22.3$ \\
Sleep latency (min) & $42.2 \pm 55.6$ \\
Sleep time below 90\% oxygen saturation (\%) & $11.9 \pm 20.3$ \\
Amount of REM (\%) & $12.8 \pm 7.8$ \\
Al score & $19.4 \pm 13.4$ \\
PLMI score & $22.0 \pm 21.5$ \\
ESS score & $5.6 \pm 5.8$ \\
Severity of OSA* & 36.4 \\
$\quad$ None (AHI < 5) & 31.8 \\
Mild (AHI 5-14.9) & 13.6 \\
Moderate (AHI 15-29.9) & 18.2 \\
Severe (AHI $\geq 30)$ & $15.8 \pm 18.6$ \\
AHI & $17.3 \pm 17.2$ \\
REM AHI
\end{tabular}

*Data is presented as percentage of patients. AHI: apnoea-hypopnoea index; Al: arousal index; ESS: Epworth Sleepiness Scale; OSA: obstructive sleep apnoea; PLMI: periodic limb movement index; REM: rapid eye movement; SD: standard deviation

Table IV. Chronic obstructive pulmonary disease severity indices.

\begin{tabular}{lc}
\hline Index & Mean \pm SD \\
\hline BODE index & $3.7 \pm 1.9$ \\
MMRC dyspnoea scale & $2.1 \pm 1.1$ \\
Distance walked in 6 min (m) & $338 \pm 77$ \\
Exacerbation rate (requiring & $0.5 \pm 0.7$ \\
hospitalisation) in the preceding year & \\
\hline
\end{tabular}

6MWD: distance walked in 6 min; BODE: body mass index, airflow obstruction, dyspnoea, and exercise; MMRC: modified Medical Research Council; SD: standard deviation 


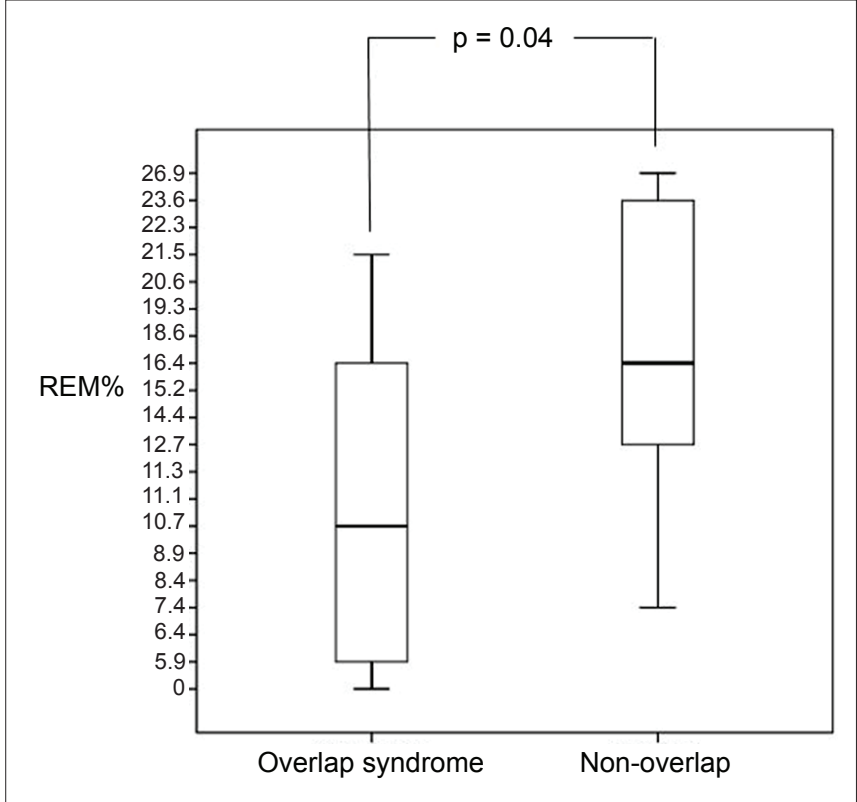

Fig. 1 Box and Whisker plot shows percentage of rapid eye movement (REM\%) sleep of patients with and without overlap syndrome. A $p$-value $<0.05$ was considered statistically significant.

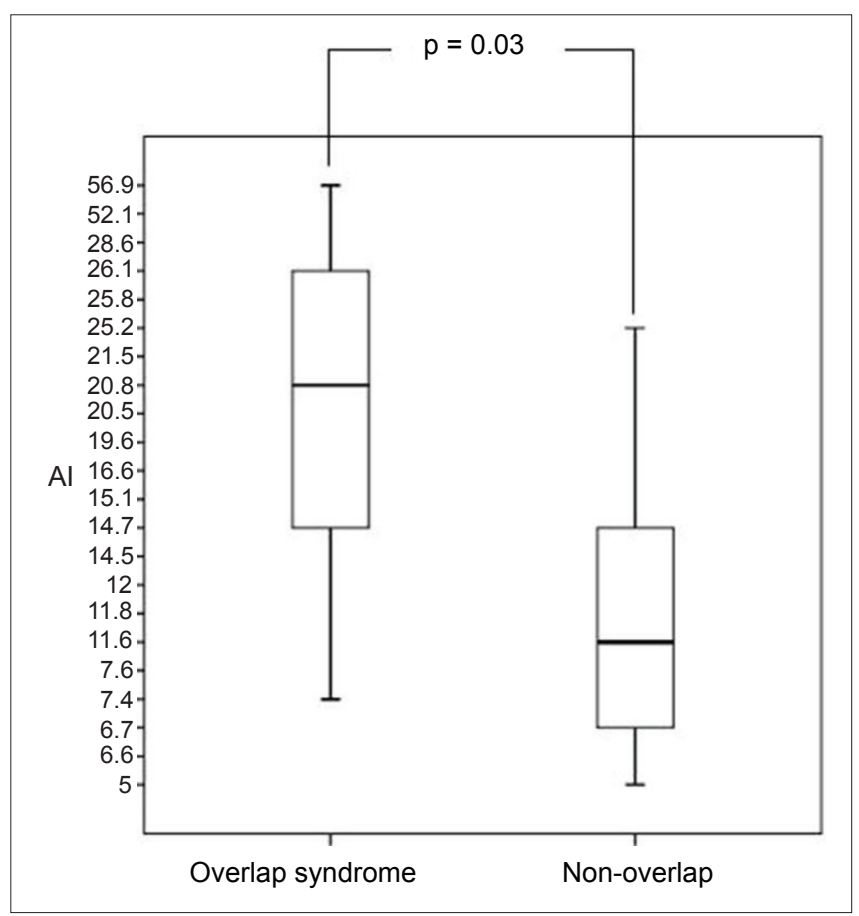

Fig. 2 Box and Whisker plot shows arousal index (AI) scores of patients with and without overlap syndrome. A p-value $<0.05$ was considered statistically significant.

high for tertiary-care COPD settings. The prevalence seen in our study is also much higher than the previously reported prevalence of COPD and SDB. ${ }^{(13,14)}$ While some earlier studies have indicated unexpectedly high prevalences of overlap syndrome, these were associated with inherent methodological biases. ${ }^{(15-18)}$ Other recent, more robust studies, however, did not find any increase in the prevalence of SDB in patients with COPD when compared to those without COPD in the general population. ${ }^{(4)}$ Sanders et al even suggested that the coexistence of COPD and SDB was more by chance than any other mechanism. ${ }^{(4)}$

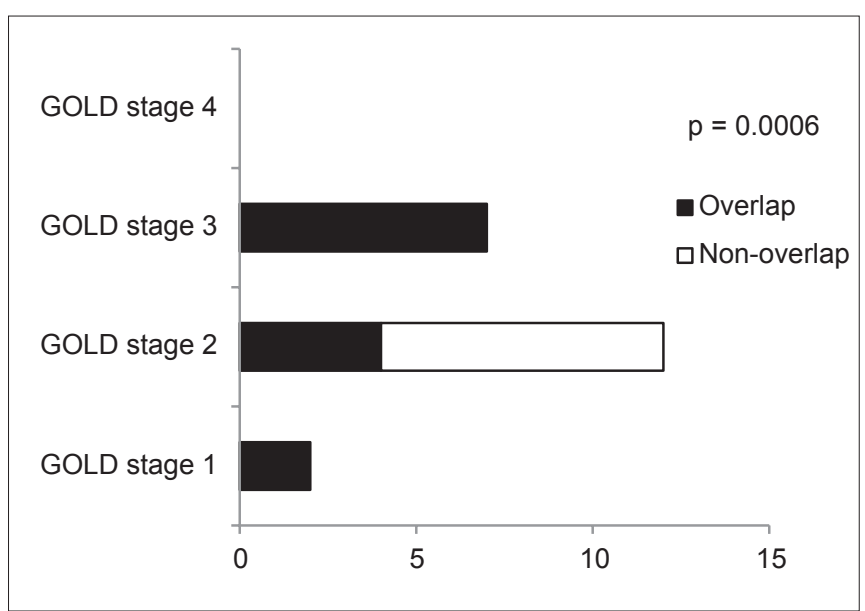

Fig. 3 Graph shows the categorisation of patients with overlap syndrome according to staging using the Global Initiative for Chronic Obstructive Lung Disease (GOLD) criteria. A p-value $<0.05$ was considered statistically significant.

Although our study population was small, there were no obvious major selection biases in our study, as all patients with COPD, irrespective of its severity, were recruited. However, the effect of potential confounders, such as diabetes mellitus or metabolic syndrome, was not accounted for in our cohort. While it is possible that with a larger study population the prevalence of overlap syndrome in settings similar to ours might be lower, this remains conjectural at present. Conversely, it is equally possible that the higher than expected prevalence of overlap syndrome in our cohort might reflect its actual incidence in the local Asian population and may be due to some as yet undiscovered aetiological factors. Additionally, there may be a possible correlation between the high incidence of overlap syndrome and the high smoking rates seen in our study cohort, as it is well known that smoking - especially heavy smoking (over 40 cigarettes/day) - is a risk factor for OSA. ${ }^{(19,20)}$ To the best of our knowledge, no previous study in the literature has specifically investigated the prevalence of overlap syndrome in an Asian population. However, it should also be noted that while our study population was Asian, it comprised a mix of the major racial groups seen in Singapore and was therefore not homogeneous.

The mean BMI of our patients was within normal limits for an Asian population. ${ }^{(21)}$ While it would be expected that mean BMI levels would be lower in our cohort than in a corresponding non-Asian population, it was surprising to find that the BMls of our patients were entirely within normal limits, considering that a majority of COPD patients in our group had OSA. The presence of COPD in our patients could have contributed to the lower mean BMI levels we observed, a phenomenon well known in patients with COPD. ${ }^{(22)}$ Another possible explanation could be the presence of craniofacial abnormalities that contributed to OSA in our cohort. We noted no significant difference in BMI between patients with overlap syndrome and those without in our study, contrary to other studies that have suggested that patients with OSA (Asian or otherwise) generally have a higher BMI than non-OSA patients. ${ }^{(23)}$ This association between BMI and OSA, however, remains debatable, with other studies reporting conflicting evidence. ${ }^{(24)}$ 
The mean predicted $\mathrm{FEV}_{1}$ percentage indicated that our cohort, as a whole, could be categorised as GOLD stage II for COPD, suggesting that our patients would have reasonable exercise tolerance. This, however, did not turn out to be true, as $6 \mathrm{MWD}$ was only $338 \pm 77 \mathrm{~m}$ in our cohort. It has been shown that $\mathrm{FEV}_{1}$ is an independent predictor of $6 \mathrm{MWD}$ in healthy populations. ${ }^{(25)}$ However, clear normative values are not available for the various GOLD stages of COPD, despite some studies indicating increasing rates of decline of $6 \mathrm{MWD}$ with increasing severity of COPD. ${ }^{(26)}$ It is unlikely that the lower 6MWD levels in our study were related to OSA, as no correlations have yet been found between OSA and 6MWD. ${ }^{(27)} 6 \mathrm{MWD}$ has been validated in previous studies, with cutoff values of $350 \mathrm{~m}$ and below reported to predict increasing mortality. ${ }^{(28)}$

The more accurate and precise BODE index for our group was $3.7 \pm 1.9$, giving a predicted 52 -month mortality of $32 \%$. ${ }^{(29)}$ The modified MRC (MMRC) dyspnoea scale score for our group suggested that the severity level of breathlessness in our patients was moderate. It is well known that the MMRC dyspnoea scale ${ }^{(30)}$ correlates poorly with spirometric measures such as $\mathrm{FEV}_{1}$, and therefore, by extension, with GOLD staging for COPD. ${ }^{(31)}$ In our patients, we found no correlation between having at least one exacerbation requiring hospitalisation in the preceding year $(40 \%$ of our group) and GOLD staging for COPD. Hurst et al reported that, with worsening lung function, the rate of exacerbation increases, ${ }^{(32)}$ although the exacerbation rate for patients with GOLD stage II COPD in that earlier study by Hurst et al was lower than the exacerbation rate of $0.5 \pm 0.7$ found in our cohort. This difference was possibly because exacerbators in the study by Hurst et al fitted the 'frequent exacerbation' phenotype the authors described. ${ }^{(32)}$ Our study was not designed to examine differences in healthcare utilisation or exacerbation rates between study patients and those with pure OSA or pure COPD alone.

Mean ESS scores were within normal limits in our cohort, which was not unusual. While ESS is a useful tool for the assessment of daytime sleepiness, it does not accurately predict the severity of sleep apnoea. ${ }^{(33)}$ Even in large databases such as the Sleep Heart Health Study (SHHS), where ESS score was found to correlate with the severity of OSA, absolute changes were small, and even the most severe OSA groups had ESS scores within normal ranges. ${ }^{(34)}$ It is also not unusual for patients with COPD to under-report sleepiness, ${ }^{(35)}$ which is often related to other predominant symptoms such as anxiety, dyspnoea, other comorbid diseases, and at later stages, marked disability.

The average $\mathrm{AHI}$ in our cohort suggests that our patients had moderate OSA, albeit with wide variation. The only two variables that were significantly different between patients with and without overlap syndrome in our study were REM\% sleep and Al scores. REM\% sleep is known to be reduced in patients with COPD, ${ }^{(36)}$ and the proportion of REM sleep is also often reduced in patients with OSA. ${ }^{(37)}$ Therefore, in our study, it is not surprising that patients with overlap syndrome had significantly reduced REM sleep than those without overlap syndrome. Objective electroencephalography evidence from many studies suggest that there is poor quality sleep in patients with COPD, with long periods of wakefulness contributed to by increased arousals. ${ }^{(38-42)}$ Similar to our study, the SHHS study also found that, among other things, patients with overlap syndrome had higher Al than patients with obstructive airways disease. ${ }^{(4)}$

Our study also showed that our study cohort, as a whole, had a significantly high PLMI, which again was not a novel finding. Other studies have demonstrated that patients with COPD show elevated PLMI, ${ }^{(43)}$ although the mechanism behind this association has yet to be established. The present study, too, was not designed to examine the relationship between periodic limb movements (PLMs) or restless legs syndrome and COPD. Nevertheless, although our patients were not specifically asked about any lower or upper limb symptoms, they did not complain about any behavioural patterns. PLMs can occur secondary to drugs, medical conditions and other sleep disorders. ${ }^{(10)}$ However, this relationship was not specifically investigated in our study. We found that all our patients without overlap syndrome were categorised as GOLD stage II for COPD. Patients in our cohort belonging to other GOLD stages all had overlap syndrome. While this finding was unusual, it was probably more a consequence of chance and related to the small sample size of our study.

The present study had some limitations. The number of patients recruited was relatively small and the majority was male. While the patient population was Asian, it was not homogeneous, with at least three different racial groups being primarily involved. These shortcomings might limit the extent to which our results can be generalised to Asian populations. We could not ascertain whether treating OSA in patients with overlap syndrome would have any impact on quality of life, lung function, exacerbations and survival, with respect to expected prognoses in the respective COPD stages. Patients with $\mathrm{BMI} \geq 30 \mathrm{~kg} / \mathrm{m}^{2}$ were excluded from our study so that the cohort was not confounded due to patients who might have $\mathrm{OHS}$, and because only patients with OSA alone were focused on. It is possible that such an exclusion could have caused an underestimation of the number of patients with overlap syndrome.

In conclusion, the prevalence of overlap syndrome was high in our Asian population, in which patients were screened and recruited from tertiary-care outpatient settings. Patients with overlap syndrome did not exhibit any daytime hypersomnolence or obese body habitus, and were impossible to distinguish clinically from patients with COPD alone, which suggests that there were no clinical predictors between the two patient groups. However, in our study, patients with overlap syndrome had significantly less REM sleep and significantly more arousals than patients with COPD. Our patients, as a whole, had elevated PLMI scores. However, the significance of this finding will need to be elucidated by further studies. Our findings suggest that it might be difficult to justify recommending polysomnography for all patients of Asian descent, irrespective of symptoms, without extensive studies in primary care and other settings. Nevertheless, our study is clinically significant, as it highlights the need for a high index of suspicion when dealing with Asian patients with overlap syndrome. Clinicians should not assume that such patients with normal BMI would be at low risk of having SDB. At the very least, our results could be used as a pilot for estimating the sample sizes of future studies. 


\section{REFERENCES}

1. Flenley DC. Sleep in chronic obstructive lung disease. Clin Chest Med 1985; 6:651-61.

2. Marin JM, Soriano JB, Carrizo SJ, Boldova A, Celli BR. Outcomes in patients with chronic obstructive pulmonary disease and obstructive sleep apnea: the overlap syndrome. Am J Respir Crit Care Med 2010; 182:325-31.

3. Weitzenblum E, Chaouat A, Kessler R, Canuet M. Overlap syndrome: obstructive sleep apnea in patients with chronic obstructive pulmonary disease. Proc Am Thorac Soc 2008; 5:237-41.

4. Sanders $\mathrm{MH}$, Newman AB, Haggerty $\mathrm{CL}$, et al; Sleep Heart Health Study. Sleep and sleep-disordered breathing in adults with predominantly mild obstructive airway disease. Am J Respir Crit Care Med 2003; 167:7-14.

5. Bednarek M, Plywaczewski R, Jonczak L, Zielinski J. There is no relationship between chronic obstructive pulmonary disease and obstructive sleep apnea syndrome: a population study. Respiration 2005; 72:142-9.

6. Rabe KF, Hurd S, Anzueto A, et al; Global Initiative for Chronic Obstructive Lung Disease. Global strategy for the diagnosis, management, and prevention of chronic obstructive pulmonary disease: GOLD executive summary. Am J Respir Crit Care Med 2007; 176:532-55.

7. Johns MW. Daytime sleepiness, snoring, and obstructive sleep apnea. The Epworth Sleepiness Scale. Chest 1993; 103:30-6.

8. Ruehland WR, Rochford PD, O'Donoghue FJ, et al. The new AASM criteria for scoring hypopneas: impact on the apnea hypopnea index. Sleep 2009 32:150-7.

9. EEG arousals: scoring rules and examples: a preliminary report from the Sleep Disorders Atlas Task Force of the American Sleep Disorders Association. Sleep 1992; 15:173-84.

10. Natarajan R. Review of periodic limb movement and restless leg syndrome. J Postgrad Med 2010; 56:157-62.

11. Celli BR, Cote CG, Marin JM, et al. The body-mass index, airflow obstruction, dyspnoea, and exercise capacity index in chronic obstructive pulmonary disease. N Engl J Med 2004; 350:1005-12.

12. Rizzi M, Palma P, Andreoli A, et al. Prevalence and clinical feature of the "overlap syndrome", obstructive sleep apnea (OSA) and chronic obstructive pulmonary disease (COPD), in OSA population. Sleep Breath 1997; 2:68-72.

13. Buist AS, McBurnie MA, Vollmer WM, et al; BOLD Collaborative Research Group. International variation in the prevalence of COPD (the BOLD Study): a population-based prevalence study. Lancet 2007; 370:741-50.

14. Young T, Palta M, Dempsey J, et al. The occurrence of sleep-disordered breathing among middle-aged adults. N Engl J Med 1993; 328:1230-5.

15. Guilleminault C, Cummiskey J, Motta J. Chronic obstructive airflow disease and sleep studies. Am Rev Respir Dis 1980; 122:397-406.

16. Chaouat A, Weitzenblum E, Krieger J, et al. Association of chronic obstructive pulmonary disease and sleep apnea syndrome. Am J Respir Crit Care Med 1995; 151:82-6.

17. Bradley TD, Rutherford A, Grossman RF, et al. Role of daytime hypoxemia in the pathogenesis of right heart failure in the obstructive sleep apnea syndrome. Am Rev Respir Dis 1985; 131:835-9.

18. Bradley TD, Rutherford A, Lue F, et al. Role of diffuse airway obstruction in the hypercapnia of obstructive sleep apnea. Am Rev Respir Dis 1986; 134:920-4.

19. Young T, Peppard PE, Gottlieb DJ. Epidemiology of obstructive sleep apnea: a population health perspective. Am J Respir Crit Care Med 2002; 165:1217-39.

20. Wetter DW, Young TB, Bidwell TR, Badr MS, Palta M. Smoking as a risk factor for sleep-disordered breathing. Arch Intern Med 1994; 154:2219-24.

21. WHO Expert Consultation. Appropriate body-mass index for Asian populations and its implications for policy and intervention strategies. Lancet 2004; 363:157-63.

22. Agustí AG. Systemic effects of chronic obstructive pulmonary disease.
Proc Am Thorac Soc 2005; 2:367-70.

23. Gami AS, Caples SM, Somers VK. Obesity and obstructive sleep apnea. Endocrinol Metab Clin North Am 2003; 32:869-94.

24. Li KK, Powell NB, Kushida C, et al. A comparison of Asian and white patients with obstructive sleep apnea syndrome. Laryngoscope 1999; 109:1937-40.

25. Camarri B, Eastwood PR, Cecins NM, Thompson PJ, Jenkins S. Six minute walk distance in healthy subjects aged 55-75 years. Respir Med 2006; 100:658-65

26. Casanova C, Cote CG, Marin JM, et al. The 6-min walking distance: longterm follow up in patients with COPD. Eur Respir J 2007; 29:535-40.

27. Alameri H, Al-Kabab Y, BaHammam A. Submaximal exercise in patients with severe obstructive sleep apnea. Sleep Breath 2010; 14:145-51.

28. Cote CG, Casanova C, Marín JM, et al. Validation and comparison of reference equations for the 6-min walk distance test. Eur Respir J 2008; 31:571-8.

29. Celli BR, Cote C, Marin JM, et al. The body-mass index, airflow obstruction, dyspnea, and exercise capacity index in chronic obstructive pulmonary disease. N Engl J Med 2004; 350:1005-12.

30. Mahler DA, Wells CK. Evaluation of clinical methods for rating dyspnea. Chest 1988; 93:580-6.

31. Bestall JC, Paul EA, Garrod R, et al. Usefulness of the Medical Research Council (MRC) dyspnoea scale as a measure of disability in patients with chronic obstructive pulmonary disease. Thorax 1999; 54:581-6.

32. Hurst JR, Vestbo J, Anzueto A, et al; Evaluation of COPD Longitudinally to Identify Predictive Surrogate Endpoints (ECLIPSE) Investigators. Susceptibility to exacerbation in chronic obstructive pulmonary disease. N Engl J Med 2010; 363:1128-38.

33. Serafini FM, MacDowell Anderson W, Rosemurgy AS, Strait T, Murr MM. Clinical predictors of sleep apnea in patients undergoing bariatric surgery. Obes Surg 2001; 11:28-31.

34. Gottlieb DJ, Whitney CW, Bonekat WH, et al. Relation of sleepiness to respiratory disturbance index: the Sleep Heart Health Study. Am J Respir Crit Care Med 1999; 159:502-7.

35. Saaresrantaa T, Irjalac K, Aittokalliod T, Polo O. Sleep quality, daytime sleepiness and fasting insulin levels in women with chronic obstructive pulmonary disease. Respir Med 2005; 99: 856-63.

36. Harding SM. Sleep in patients with pulmonary disease. In: Pagel JF, PandiPerumal SR, eds. Primary Care Sleep Medicine: A Practical Guide. Totowa: Humana Press Inc, 2007: 171-89.

37. Patil SP, Schneider H, Schwartz AR, Smith PL. Adult obstructive sleep apnea: pathophysiology and diagnosis. Chest 2007; 132:325-37.

38. Leitch AG, Clancy LJ, Leggett RJ, et al. Arterial blood gas tensions, hydrogen ion, and electroencephalogram during sleep in patients with chronic ventilatory failure. Thorax 1976; 31:730-5

39. Arand DL, McGinty DJ, Littner MR. Respiratory patterns associated with hemoglobin desaturation during sleep in chronic obstructive pulmonary disease. Chest 1981; 80:183-90.

40. Fleetham J, West P, Mezon B, et al. Sleep, arousals, and oxygen desaturation in chronic obstructive pulmonary disease. The effect of oxygen therapy. Am Rev Respir Dis 1982; 126:429-33.

41. Calverley PM, Brezinova V, Douglas NJ, Catterall JR, Flenley DC. The effect of oxygenation on sleep quality in chronic bronchitis and emphysema. Am Rev Respir Dis 1982; 126:206-10.

42. Cormick W, Olson LG, Hensley MJ, Saunders NA. Nocturnal hypoxaemia and quality of sleep in patients with chronic obstructive lung disease. Thorax 1986; 41:846-54.

43. Charokopos N, Leotsinidis M, Pouli A, et al. Periodic limb movement during sleep and chronic obstructive pulmonary disease. Sleep Breath 2008; 12:155-9. 